\title{
Validierung einer Skala zur Erfassung der kollektiv-moralischen Einstellung in Sportgruppen (KMES)
}

\author{
Theresa Manges (1), Nadja Walter und Anne-Marie Elbe
}

Institut für Sportpsychologie und Sportpädagogik, Sportwissenschaftliche Fakultät, Universität Leipzig

\begin{abstract}
Zusammenfassung: Der vorliegende Beitrag stellt eine Skala zur Erhebung der kollektiv-moralischen Einstellung einer Gruppe für den deutschsprachigen Sport- und insbesondere Dopingkontext vor und überprüft sie hinsichtlich ihrer Testgütekriterien. Die KMES wurde auf der Grundlage der Collective Moral Motivation Scale aus der englischsprachigen Arbeits- und Organisationspsychologie entwickelt und anschließend anhand einer Gesamtstichprobe von 545 Personen (Substichprobe 1 und 3: Nachwuchsleistungsathlet_innen; Substichprobe 2: Sportstudierende) validiert. Eine konfirmatorische Faktorenanalyse bestätigt das postulierte eindimensionale Konstrukt, welches eine exzellente interne Konsistenz und eine akzeptable Retest-Reliabilität aufweist. Darüber hinaus zeigt die KMES Kriteriumsvalidität, indem sie die Außenkriterien antisoziales Verhalten und Dopingabsichten vorhersagen kann sowie erwartungskonforme Zusammenhänge zum motivationalen Trainingsklima abbildet. Die Ergebnisse deuten darauf hin, dass die KMES zur Erfassung von Werten auf kollektiver Ebene eingesetzt werden kann. Dies könnte vor allem in Anti-Doping-Studien neue Einblicke generieren.
\end{abstract}

Schlüsselwörter: moralische Einstellung, Sportgruppe, Testgütekriterien

Validation of the KMES Scale for Assessing the Collective Moral Attitude of Sport Groups

Abstract: The present study introduces an instrument to measure the collective moral attitude of a group in the sports and doping context and evaluates its psychometric quality. The KMES scale was developed based on the Collective Moral Motivation Scale, which is used in English-speaking organizational psychology. It was then validated through a sample of $N=545$ subjects, consisting of young elite athletes (subsamples 1 and 3) and sport students (subsample 2). Confirmatory factor analysis confirms the postulated one-dimensional structure of the KMES. Further analyses indicate excellent internal consistency and acceptable retest reliability. Moreover, the scale demonstrates criterion validity since it was able to predict antisocial behavior as well as doping intentions. In line with our expectations, the KMES correlated with motivational climate and therefore additionally proves its validity. These results suggest that the KMES is a suitable instrument for measuring values on a collective group level. It could generate new insights especially in antidoping studies.

Keywords: moral attitude, sport group, psychometric quality

Sport gilt als ein idealer Kontext für die Förderung moralischer Kompetenzen und ethischer Verhaltensweisen eines Individuums (Kavussanu, Roberts \& Ntoumanis, 2002). Alltäglich sind Sportler_innen jedoch Situationen ausgesetzt, in denen sie sich entscheiden müssen, ob sie sich den grundlegenden Werten des Sports entsprechend verhalten oder diese, z. B. um die eigenen Gewinnchancen zu erhöhen, vernachlässigen. Ein Verhalten wird als unmoralisch und unethisch angesehen, wenn es gegen die geltenden Normen und Werte wie Fairplay, Ehrlichkeit oder Respekt gegenüber den Regeln verstößt. In der Tat wird aus der Sportwelt regelmäßig von Fouls, Beschimpfungen, Betrug und Täuschung berichtet (Kavussanu \& Boardley, 2009). Neben antisozialem Verhalten ist Doping als einer der schwerwiegendsten Fälle von Betrug im Sport ein zentraler Gegenstand der Erforschung moralre- levanten Verhaltens. Betrachtet man die Faktoren, die mit transgressiven Verhaltensweisen und Verhaltensabsichten assoziiert sind, ist Folgendes festzustellen: Erstens spielen moralische Variablen eine entscheidende Rolle in Bezug auf Doping (z.B. Ntoumanis, Ng, Barkoukis und Backhouse, 2014) und antisoziales Verhalten (z.B. Kavussanu, Stanger und Boardley, 2013); zweitens muss zur Erklärung solcher Verhaltensweisen- und Absichten das soziale Umfeld von Athlet_innen berücksichtigt werden, wie Modelle aus der Dopingforschung, z.B. das Sport Drug Control Model (SDCM; Donovan, Egger, Kapernick \& Mendoza, 2002) und das Life Cycle Model (LCM; Petroczi \& Aidman, 2008) unterstreichen. In beiden Modellen sind systemische Faktoren wie Teamnormen von zentraler Bedeutung und, wie nachfolgend dargestellt, belegen zahlreiche weitere Studien, dass kollektive Nor- 
men und Werte eines Teams oder einer Trainingsgruppe mit den moralischen Einstellungen und Verhaltensweisen der einzelnen Gruppenmitglieder zusammenhängen. Dies ist im Einklang mit der Theorie des geplanten Verhaltens (Ajzen, 1985), die besagt, dass subjektive Normen, zusammen mit persönlichen Einstellungen und der wahrgenommenen Verhaltenskontrolle, die Verhaltensabsichten und das tatsächliche Verhalten einer Person formen. Aus diesem Grund ist es notwendig, zur Untersuchung unmoralischen Verhaltens im Sport die kollektiv-moralische Einstellung standardisiert erfassen zu können. Dem Bedarf eines einheitlichen, reliablen und validen Instrumentes, welches im Kontext Sport moralische Normen und Werte auf kollektiver Ebene erhebt, versucht der vorliegende Beitrag nachzukommen, indem die Skala zur Erfassung der kollektiv-moralischen Einstellung in Sportgruppen (KMES) vorgestellt und validiert wird. Wir bedienen uns dabei einer für diesen Zweck vielversprechenden Skala aus der englischsprachigen Arbeits- und Organisationspsychologie, der Collective Moral Motivation Scale (CMMS; Arnaud, 2010), und adaptieren diese auf den Sportgruppenkontext sowie auf die deutsche Sprache.

\section{Theoretischer Hintergrund}

Neben der Theorie des geplanten Verhaltens (Ajzen, 1985) wird zur Erklärung von unethischem, antisozialem Verhalten, und insbesondere Dopingverhalten oftmals die sozial-kognitive Theorie Banduras (1991) herangezogen (z. B. Boardley, Grix, \& Dewar, 2014; Zelli, Mallia \& Lucidi, 2010). Sie sagt aus, dass moralisches Verhalten durch moralische Standards gesteuert wird, welche wiederum durch Sozialisationsprozesse entwickelt und internalisiert wurden. Genauer gesagt, wird die Entscheidung zu dopen, zu foulen oder sich anderweitig transgressiv zu verhalten, durch das Zusammenspiel von persönlichen Faktoren und sozialen bzw. Umfeldfaktoren bestimmt. Den sozialen Kontext, in dem moralische Entscheidungen im Sport stattfinden, bildet in erster Linie das Team oder die Trainingsgruppe von Sportler_innern. Sowohl die Trainer_innen als auch die Teamkamerad_innen tragen nachweislich dazu bei, die Einstellungen und Entscheidungen, die Motivation und das Verhalten einer Person im sportlichen Kontext $\mathrm{zu}$ beeinflussen (Ntoumanis, Taylor \& Thøgersen-Ntoumani, 2012). Bei der Erforschung des Einflusses der Trainingsgruppe auf individuelle Einstellungen und Verhaltensweisen, stellen das motivationale Trainingsklima und die moralische Atmosphäre/die moralischen Gruppennormen die zentralen Größen dar. Die Moralische Atmosphäre bezieht sich auf die kollektiven
Gruppennormen bezüglich moralischen Handelns und definiert somit, was in der Gruppe als akzeptables Verhalten angesehen wird (Power, Higgins \& Kohlberg, 1989). Eine Vielzahl an Studien untersuchte dieses Konstrukt in Bezug auf moralrelevantes Verhalten im Sportkontext, wobei jedoch verschiedene Begriffe und unterschiedliche Methoden zur Erfassung des Konstruktes der kollektiven Gruppennormen verwendet wurden. Stephens (2000, 2001, 2004) und Guivernau und Duda (2002), beispielsweise, nannten das interessierende Konstrukt moralische Atmosphäre und untersuchten diese, in dem sie die Athlet_innen baten, anzugeben, wie viele der Gruppenmitglieder in einer hypothetischen Situation Gegner_innen verletzen würden und wie die Trainer_innen dazu stehen würde. Die Ergebnisse offenbarten, dass Sportler_innen, die die Normen in ihrem Team pro-aggressiv wahrnahmen, auch eher dazu neigten, sich selbst aggressiv zu verhalten. Dies geht mit den Befunden einer aktuellen Metaanalyse von Spruit, Kavussanu, Smit und IJntema (2019) einher, in welcher der Begriff moralisches Klima verwendet wurde und die bestätigt, dass das moralische Verhalten von Nachwuchssportler_innen mit dem vorherrschenden moralischen Klima des Trainingsumfeldes korreliert ist. Über die 27 in die Metaanalyse einbezogenen Studien $(N=7.726)$ hinweg war das prosoziale Verhalten von Athlet_innen stärker und das antisoziale Verhalten schwächer ausgeprägt, wenn ein hohes moralisches Klima im Team herrschte. Allerdings konnte für einige der eingeschlossenen Studien nicht nachvollzogen werden, welche Methode zur Erfassung des moralischen Klimas gewählt wurde. Für einen Großteil der Studien (z.B. Kavussanu, Roberts \& Ntoumanis, 2002) kamen unterschiedliche, meist für die jeweilige Studie modifizierte Versionen des Team Norm Questionnaire (TNQ; Shields, Bredemeier, Gardner \& Bostrom, 1995) zum Einsatz. Wie oben beschrieben, machen die Proband_innen darin Angaben über die geschätzte Anzahl an Teamkamerad_innen, die sich in einer hypothetischen Situation, in der es um das Gewinnen geht, unfair, aggressiv oder antisozial verhalten würden sowie Angaben darüber, ob die Trainer_innen ein solches Verhalten befürworten würde. Der TNQ diente auch als Grundlage zur Messung der moralischen Atmosphäre in Bezug auf Dopingabsichten (Kavussanu, Elbe \& Hatzigeorgiadis, 2015) und wurde hierfür auf einen dopingspezifischen Kontext umformuliert. Es zeigte sich, dass Sportler_innen höhere Dopingabsichten angaben, wenn sie eine wenig ausgeprägte moralische Atmosphäre in der Trainingsgruppe wahrnahmen, das heißt die Teamnormen Doping befürworteten. Darüber hinaus berichteten Ntoumanis et al. (2014), dass wahrgenommene soziale und moralische Normen des Trainingsumfeldes starke Indikatoren für Dopingabsichten in 11 Studien $(N=5.000)$ darstellten. Allerdings wur- 
den in einigen der einbezogenen Studien die wahrgenommenen Normen von signifikanten Personen im Allgemeinen abgefragt, sodass kein Rückschluss darauf möglich war, welche Personen oder Personengruppen gemeint waren (z. B. „most people who are important to me would want me to use prohibited substances to improve my performance“, Lazuras, Barkoukis, Mallia, Lucidi \& Brand, 2017, S. 199). Mit dieser Messung lässt sich deshalb nicht genau feststellen, ob sich die wahrgenommenen Normen tatsächlich auf die Trainingsgruppe oder eher auf Eltern, Geschwister, Freunde oder andere Personen beziehen. Zusammenfassend zeigt die Studienlage Evidenz dafür, dass kollektive moralische Normen des Teams oder der Trainingsgruppe, mögen diese als moralische Atmosphäre, moralisches Klima oder moralische Normen bezeichnet worden sein, einen Einfluss auf vielerlei unmoralisches Verhalten und Verhaltensabsichten besitzen, weshalb antisoziales Verhalten und Dopingabsichten in der vorliegenden Arbeit als Kriterien für die Validitätsprüfung herangezogen werden.

Neben den in der Gruppe geltenden moralischen Normen ist das motivationale Trainingsklima einer Gruppe ein weiteres viel untersuchtes soziales Konstrukt in Bezug auf Doping und antisoziales Verhalten. Es beschreibt die, meist von Trainer_innen induzierten, Leistungsziele und Erfolgskriterien und die damit vermittelten Werte in der Gruppe (Ames, 1992). Dabei kann es zwei Tendenzen annehmen (Seifriz, Duda und Chi, 1992): In einem wettbewerbsorientierten Klima liegt der Schwerpunkt auf dem Übertreffen anderer, dem Gewinnen und der hauptsächlichen Beachtung der Besten. Dagegen liegt ein aufgabenorientiertes Klima vor, wenn der individuelle Fortschritt und die Entwicklung der Fertigkeiten jedes einzelnen Mitglieds von zentraler Bedeutung sind. In verschiedenen Forschungsarbeiten konnte ein Zusammenhang zwischen dem motivationalen Klima eines Teams und dem ethisch-moralischen Verhalten der Teammitglieder festgestellt werden (z. B. Stanger, Backhouse, Jennings \& McKenna, 2018; van de Pol, Kavussanu \& Claessens, 2020). Kavussanu et al. (2020) berichten zudem, dass ein wettbewerbsorientiertes motivationales Klima, in dem das Gewinnen und Übertreffen anderer im Vordergrund steht, die Dopingwahrscheinlichkeit positiv vorhersagen konnte. Darüber hinaus haben Forschungsergebnisse gezeigt, dass das motivationale Trainingsklima mit den wahrgenommenen moralischen Gruppennormen zusammenhängt. In einer Reihe von Studien konnten moderate Korrelationen zwischen beiden Konstrukten nachgewiesen werden (z. B. Kavussanu \& Spray, 2006; Kavussanu et al., 2002; Ommundsen, Roberts, Lemyre \& Treasure, 2003) und zwar in der Art, dass ein wettbewerbsorientiertes Trainingsklima wahrgenommen wurde, wenn die moralische Atmosphäre/die Gruppennormen unfaires
Verhalten und Aggression duldeten oder befürworteten. Ein aufgabenorientiertes Klima wurde dagegen mit der Wahrnehmung verbunden, dass die geltenden Normen kein unmoralisches Verhalten zuließen. Aufgrund der Persistenz dieser Beziehung über Geschlecht, Sportarten und kulturellen Kontext hinweg (Kavussanu \& Spray, 2006) wird das motivationale Trainingsklima in der vorliegenden Arbeit als weiteres Kriterium zur Validierung herangezogen.

Trotz der aufgeführten Befunde mangelt es im deutschsprachigen Raum an einem geeigneten Instrument, das im Sport- und insbesondere Dopingkontext zur Messung kollektiver moralischer Normen und Werte eingesetzt werden kann. Um dieses Desiderat aufzugreifen, lohnt ein Blick in die Nachbardisziplin der Arbeits- und Organisationspsychologie. Arnaud (2010) präsentiert ein Instrument, welches die kollektiven Gruppennormen erschließt, indem moralische Werte potentiell konkurrierenden Werten gegenüber gestellt werden und nach der in der Gruppe wahrgenommenen Priorisierung gefragt wird. Diese Erfassung erlaubt ein Gesamtbild der in der Gruppe vorherrschenden Normen und Werte, während Messmethoden wie der TNQ (Shields et al., 1995) und Skalen für subjektive Normen (z. B. Lazuras et al., 2017) in der Regel die Normen gegenüber einem bestimmten Verhalten (z. B. Foulen oder Dopen) abbilden.

\section{Die Collective Moral Motivation Scale (CMMS)}

Für die vorliegende Studie wurde daher als Grundlage die CMMS aus dem arbeits- und organisationspsychologischen Kontext herangezogen. Die Skala erhebt die von den Mitarbeitenden wahrgenommene kollektive moralische Motivation der entsprechenden Firma oder Abteilung. Sie ist Teil des Ethical Climate Index (ECI; Arnaud, 2010), welcher das ethische Arbeitsklima entlang der vier Dimensionen Collective Moral Sensitivity, Collective Moral Judgement, Collective Moral Character und Collective Moral Motivation bestimmt. Letztere untersucht die vorherrschenden Werte und Normen, die innerhalb eines sozialen Systems (z.B. einer Abteilung) existieren. Konkret beschreibt die Skala das von den Mitgliedern des Systems wahrgenommene Ausmaß darüber, welche Priorität moralische Werte wie Ehrlichkeit, Fairness oder Hilfsbereitschaft gegenüber anderen Werten wie Macht, Leistung oder persönlichem Erfolg in dem System besitzen. Damit gibt sie Auskunft darüber, inwiefern die entsprechende Gruppe moralisch motiviert bzw. eingestellt ist. 
Die Grundlage für diese Skala bildete die Werttheorie von Schwartz (Theory of Basic Values, 1992), die auch zunehmend in der sportpsychologischen Forschung Anwendung findet (Ring, Kavussanu \& Gürpınar, 2020). Laut Schwartz $(1992,2012)$ dienen Werte als Leitprinzipien, die nach Priorität geordnet sind und somit das Verhalten und die Entscheidungen von Personen und Gruppen maßgeblich lenken. Personen oder Gruppen, die moralisch eingestellt bzw. moralisch motiviert sind, werden dementsprechend moralische Werte gegenüber anderen Werten priorisieren (Arnaud, 2010). In der CMMS werden Personen mit gegensätzlichen Werten konfrontiert und gebeten, anzugeben, welche der Werte in ihrer $\mathrm{Ab}$ teilung die höhere Prävalenz und Priorität besitzen. Aufbauend auf den von Schwartz (1992) identifizierten Basiskategorien an Werten wurden die acht Items der CMMS konzipiert. Beispielsweise entsprechen die Basiskategorien Universalism und Benevolence typischen ethischen Werten wie Ehrlichkeit und sozialer Gerechtigkeit und werden den Wertekategorien Power und Achievement gegenübergestellt. So entstanden Itemformulierungen wie "Around here, power is more important than honesty" oder „In my department personal success is more important than helping others". Die Items werden auf einer fünfstufigen Likertskala von 1 „Describes my department very well“ bis 5 ,Does not describe my department at all“ eingeschätzt. Als Skalenwert dient das arithmetische Mittel, wobei höhere Werte bedeuten, dass die Gruppe als moralisch eingestellt wahrgenommen wird, bzw. moralische Gruppennormen wahrgenommen werden. Arnaud (2010) berichtet für die CMMS eine interne Konsistenz von $\alpha=.89$. Neben dem Nachweis der konvergenten und diskriminanten Validität konnte Arnaud (2010) Kriteriumsvalidität demonstrieren, indem sich die CMMS als signifikanter Prädiktor für ethisches bzw. moralisches Verhalten im Arbeitskontext erwies.

\section{Zielstellung}

Ziel der vorliegenden Studie ist es, auf der Grundlage der CMMS (Arnaud, 2010) ein Instrument zur Erhebung der prävalenten Werte und Normen einer Gruppe für den deutschsprachigen Sport- und insbesondere den Dopingkontext zu erstellen und dieses Instrument hinsichtlich seiner Testgütekriterien zu überprüfen. Dafür wird die CMMS zunächst in die deutsche Sprache übertragen und gleichzeitig für den Sportgruppen-Kontext adaptiert. Der resultierende Fragebogen wird „Skala zur Erfassung der kollektiv-moralischen Einstellung in Sportgruppen " (KMES) genannt. Der Name weicht bewusst etwas von dem Namen der zugrunde liegenden CMMS ab, da sich der Begriff „Motivation“ in diesem Zusammenhang auf die Einstellung bezieht, und nicht etwa auf den Antrieb für eine bestimmte Tätigkeit, wie mit dem Begriff Motivation oftmals assoziiert.

Der adaptierte Fragebogen KMES wird anschließend mittels einer konfirmatorischen Faktorenanalyse auf faktorielle Validität hin untersucht. Es wird, wie in der englischen, arbeitsspezifischen Version, ein eindimensionales Konstrukt angenommen. Weiterhin wird die Reliabilität und die Retest-Reliabilität der Skala sowie die Trennschärfe der Items geprüft. Bezüglich der Kriteriumsvalidität (konkurrente Validität) wird angenommen, dass die kollektiv-moralische Einstellung antisoziales Verhalten (z. B. Spruit et al., 2019) und Dopingabsichten (z. B. Kavussanu et al., 2015) vorhersagen kann. Es werden jeweils negative lineare Zusammenhänge erwartet. Weiterhin werden Zusammenhänge zwischen der KMES und dem motivationalen Trainingsklima betrachtet. Wie im theoretischen Teil erläutert, sollte eine hohe moralische Gruppeneinstellung mit einem geringen wettbewerbsorientierten Klima und einem hohen aufgabenorientierten Klima korrelieren (Kavussanu \& Spray, 2006).

\section{Methode}

\section{Stichproben und Untersuchungsdurchführung}

Die Datensätze von drei verschiedenen Substichproben (siehe Tab. 1) bildeten die Grundlage für die vorliegende Untersuchung. Insgesamt wurden $N=545$ Sportler_innen mit einem Durchschnittsalter von 18 Jahren $(S D=4.0)$ befragt. Von ihnen waren $45.4 \%$ weiblichen und $54.6 \%$ männlichen Geschlechts. Die Athlet_innen waren im Mittel seit 9.1 Jahren $(S D=4.1)$ in verschiedenen Mannschaftssportarten (32.7\%; z. B. Basketball, Rugby, Fußball, Hockey) und Individualsportarten (67.3\%; z. B. Judo, Radsport, Biathlon, Schwimmen) aktiv. Nahezu alle Teilnehmenden (99\%) machten Angaben zu ihrem Wettkampfniveau, wobei sich $22.6 \%$ dem Freizeitsport, $13.7 \%$ dem regionalen, $43.3 \%$ dem nationalen und $19.4 \%$ dem internationalen Leistungssport zuordneten. Informationen zur Wettkampfhäufigkeit machten $99.2 \%$ der Befragten. Davon nahmen $44.4 \%$ immer, $37.3 \%$ oft, $10.7 \%$ selten und $6.8 \%$ nie an Wettkämpfen teil.

Die Daten der drei Substichproben wurden folgendermaßen erhoben: Für die Stichprobe $1(n=267)$ wurde der Papierfragebogen im Zeitraum von Juni bis September 2019 über instruierte Lehrpersonen einer Eliteschule des Sports an jugendliche Leistungssport- 
Tabelle 1. Übersicht der drei Substichproben

\begin{tabular}{|c|c|c|c|c|c|}
\hline & & Erhebung 1 & Erhebung 2 & Erhebung 3 & Gesamt \\
\hline$N$ & & 267 & 179 & 99 & 545 \\
\hline Rekrutierung & & Sportschulen & Universität & Sportschulen & \\
\hline Erfasste Skalen & & $\begin{array}{c}\text { KMES, } \\
\text { Dopingabsichten }\end{array}$ & $\begin{array}{c}\text { KMES + Retest, } \\
\text { PMCSQ-D, PABSS-D }\end{array}$ & KMES & \\
\hline \multirow[t]{2}{*}{ Geschlecht (in \%) } & q & 45.7 & 46.4 & 43.4 & 45.4 \\
\hline & $\hat{0}$ & 54.3 & 53.6 & 56.6 & 54.6 \\
\hline Alter & $(M ; S D)$ & $16.4(1.8)$ & $21.9(4.4)$ & $14.8(1.6)$ & $18.0(4.0)$ \\
\hline \multirow[t]{2}{*}{ Sportart (in \%) } & MS & 32.2 & 46.4 & 5.9 & 32.7 \\
\hline & IS & 67.8 & 53.6 & 94.1 & 67.3 \\
\hline \multirow[t]{5}{*}{ Wettkampfniveau (in \%) } & FS & 3.4 & 61.5 & 1.0 & 22.6 \\
\hline & LSR & 8.6 & 25.7 & 6.1 & 13.7 \\
\hline & LSN & 55.1 & 9.5 & 71.7 & 43.3 \\
\hline & LSI & 30.3 & 3.4 & 20.2 & 19.4 \\
\hline & $\mathrm{F}$ & 2.6 & 0.0 & 1.0 & 1.0 \\
\hline \multirow[t]{5}{*}{ Wettkampfhäufigkeit (in \%) } & $\mathrm{N}$ & 0.7 & 19.0 & 0.0 & 6.8 \\
\hline & S & 4.5 & 24.0 & 2.0 & 10.7 \\
\hline & 0 & 36.3 & 36.3 & 39.4 & 37.3 \\
\hline & 1 & 56.9 & 20.7 & 58.6 & 44.4 \\
\hline & $\mathrm{F}$ & 1.1 & 0.0 & 0.0 & 0.8 \\
\hline Jahre im Sport (M; SD) & & $8.4(2.8)$ & $10.7(5.5)$ & $8.2(2.8)$ & $9.1(4.1)$ \\
\hline
\end{tabular}

Anmerkungen: Folgende Angaben in \%: F = Fehlende Werte, MS = Mannschaftssport, IS = Individualsport, FS = Freizeitsport, LSR = Leistungssport regional, $\mathrm{LSN}=$ Leistungssport national, $\mathrm{LSI}=$ Leistungssport international, $\mathrm{N}=\mathrm{Nie}, \mathrm{S}=$ Selten, $\mathrm{O}=\mathrm{Oft}, \mathrm{I}=\mathrm{Immer}$

ler_innen zum Ausfüllen verteilt. Der Fragebogen umfasste, neben demographischen Daten und der KMES zwei hypothetische Dopingszenarien zur Erfassung von Dopingabsichten. Für die Stichprobe $2(n=179)$ wurde ein Online-Fragebogen über Unipark programmiert und an der Universität über verschiedene Lehrveranstaltungen an Sportstudent_innen gestreut. Die Studierenden füllten im Oktober 2019 die Testbatterie online aus, die sich aus den folgenden Instrumenten zusammensetzte: KMES, PMCSQ-D, Prosocial and Antisocial Behavior in Sport Scale (PABSS-D) sowie demographische Daten. Von dieser Stichprobe füllten 63 Personen nach 6 bis 8 Wochen die KMES erneut aus, um die Retest-Reliabilität bestimmen zu können. Für die Stichprobe $3(n=99)$ wurde erneut der Papierfragebogen verwendet und im September 2020 an verschiedenen Sportschulen über instruierte Mitarbeitende der Universität zum Ausfüllen an die Schüler_innen verteilt. Der Fragebogen umfasste demographische Daten sowie die KMES. Ein Überblick über die deskriptiven Merkmale der Substichproben sowie deren Rekrutierungsart und die jeweils erfassten Skalen ist in Tabelle 1 dargestellt. Die Untersuchungsdurchführung wurde vom Ethikbeirat der Universität genehmigt und es wurde die informierte Einwilligung der Versuchspersonen bzw. bei minderjährigen Teilnehmenden (Sub- stichprobe 1 und 3) die Einverständniserklärung der Erziehungsberechtigten eingeholt.

\section{Instrumente}

\section{Kollektiv-moralische Einstellung einer Sportgruppe}

Zur Erhebung der kollektiv-moralischen Einstellung eines Teams oder einer Trainingsgruppe wurde die Skala KMES eingesetzt. Diese wurde im Rahmen der vorliegenden Studie in Anlehnung an die Subskala Collective Moral Motivation des Ethical Climate Index (Arnaud, 2010) entwickelt. Dazu wurden die acht Items der Skala aus dem Englischen übersetzt und gleichzeitig aus dem Arbeits- und Organisationskontext für den Sportkontext adaptiert. Gemäß den Empfehlungen einer kollaborativen Vorgehensweise (Harkness, 2003) fertigten im ersten Schritt drei Personen individuell sowie eine Gruppe von Studierenden zusammen, jeweils eine Version der Skala an, sodass vier adaptierte Versionen entstanden. Die verschiedenen Versionen wurden anschließend in einer Review-Sitzung diskutiert und die besten Itemformulierungen in Hinblick auf deren Bedeutung gemäß der zugrundeliegenden Theorie (Schwartz, 1992) für eine vorläufige Endversion zusammengetragen. Diese Endversion wurde im Rahmen einer Pilotstudie mit studentischen Teilneh- 
menden getestet und daraufhin zur Finalversion optimiert. Die finalen Itemformulierungen sind in Tabelle 2 dargestellt. Der Grad der Zustimmung bzw. Ablehnung wurde auf einer fünfstufigen Likertskala von 1 ,voll und ganz“ bis 5 ,überhaupt nicht“ ausgedrückt. Zur Bildung des Gesamtskalenwertes wurde das arithmetische Mittel über die Items gebildet, wobei höhere Werte für eine höhere kollektiv-moralische Einstellung stehen.

\section{Dopingabsichten}

Die Dopingabsichten der Teilnehmenden wurden mittels zwei Dopingszenarien, formuliert in Anlehnung an die Studie von Kavussanu et al. (2015), erhoben. Diese Szenarien beschreiben hypothetische Situationen, in denen Sportler_innen versucht sein könnten, zu dopen, um ihre Leistung zu steigern (Szenario 1) oder sich schneller von einer Verletzung zu erholen (Szenario 2). Die Teilnehmenden wurden gebeten, zu jedem Szenario die folgenden drei Fragen zu beantworten: (a) „Wie wahrscheinlich ist es, dass du den verbotenen Wirkstoff einnimmst?", (b) „Wie groß wäre die Versuchung, den verbotenen Wirkstoff einzunehmen?“ und (c) „Inwieweit wärst du dazu bereit, den verbotenen Wirkstoff einzunehmen?". Alle Fragen wurden auf einer siebenstufigen Likertskala von 1 (a) „sehr unwahrscheinlich“, (b) „sehr gering“ und (c) „überhaupt nicht bereit“ bis 7 (a) „sehr wahrscheinlich“, (b) „sehr hoch“ und (c) „sehr bereit" beantwortet. Ein Gesamtwert für Dopingabsichten wurde aus dem arithmetischen Mittel aller 6 Items berechnet. Ein höherer Wert spricht für höhere Dopingabsichten. Die interne Konsistenz fiel in der vorliegenden Untersuchung mit $\alpha=$ .88 hoch aus.

\section{Antisoziales Verhalten}

Die Messung dieses Verhaltenskriteriums erfolgte mit der deutschsprachigen Prosocial and Antisocial Behavior in Sport Scale (PABSS-D, Sohnsmeyer, 2018), deren englische Originalversion von Kavussanu und Boardley (2009) entwickelt wurde. Der Gesamtfragebogen besteht aus 20 Items, die pro- und antisoziales Verhalten gegenüber Mitspieler_innen und Gegenspieler_innen erfassen. Für die vorliegende Untersuchung wurden die 13 Items der Subskala "antisoziales Verhalten“ herangezogen. Alle Items werden mit den Worten „In der letzten Saison habe ich..." eingeleitet und zum Beispiel mit „versucht einen Gegenspieler zu verletzen." ergänzt. Die Versuchspersonen antworteten auf einer fünfstufigen Likertskala von 1 „nie“ bis 5 ,sehr oft“. Zur Bildung des Skalenwertes wurde das arithmetische Mittel über die 13 Items berechnet, wobei ein hoher Wert eine hohe Ausprägung von antisozialem Verhalten bedeutet. Die Skalenhomogenität für antisoziales Verhalten erwies sich für die vorliegende Studie als exzellent $(\alpha=.91)$.

\section{Motivationales Trainingsklima}

Zur Erhebung des motivationalen Trainingsklimas fand die deutsche Version des Perceived Motivational Climate in Sports Questionnaire (PMCSQ-D, Würth, Saborowski \& Alfermann, 1999) Anwendung. Die englischsprachige Originalversion wurde von Seifriz, Duda und Chi (1992) entwickelt. Das Instrument besteht aus den zwei Subskalen aufgabenorientiertes Klima (AUF; 6 Items) und wettbewerbsorientiertes Klima (WETT; 9 Items), deren Items alle mit dem Stamm „In meiner Trainingsgruppe...“ beginnen. Eine Fortsetzung lautet zum Beispiel „trainieren wir hart, weil wir Neues lernen wollen“ (AUF) oder ,,ist es wichtig, besser als andere zu sein" (WETT). Die Items wurden auf einer fünfstufigen Likertskala von 1 ,trifft überhaupt nicht zu“ bis 5 ,trifft voll und ganz zu“ beantwortet. Zur Bildung der zwei Subskalen wurde das arithmetische Mittel über die zugehörigen Items berechnet. Reliabilitätsanalysen zeigten in der vorliegenden Studie akzeptable (AUF $\alpha=$ .75) bis hohe (WETT $\alpha=.83$ ) interne Konsistenzen für die Subskalen auf.

\section{Datenanalyse}

Die erhobenen Daten wurden mithilfe von IBM SPSS Statistics 27.0 ausgewertet. Um die Verteilung der Items der KMES zu beurteilen, dienten die absoluten Werte von Schiefe und Kurtosis, da diese Werte bei größeren Stichproben $(N>200)$ entscheidender sind als die statistische Signifikanz (Kline, 2005). Dabei sollte die Schiefe Werte < 2 und die Kurtosis Werte $<7$ annehmen (Bühner, 2011). Weiterhin wurden die Items hinsichtlich ihrer Trennschärfe untersucht. Hierbei gelten Trennschärfekoeffizienten $r_{\text {it }}>.30$ als akzeptabel und $>.50$ als hoch (Bühner, 2011). Zur Bestimmung der internen Konsistenz der Skala wurde Cronbachs Alpha herangezogen. Die Retest-Reliabilität wurde durch die Berechnung des Pearson-Korrelationskoeffizienten $r_{\mathrm{tt}}$ zwischen zwei Erhebungszeitpunkten ermittelt. Um zu überprüfen, ob die KMES ebenso wie die zugrundeliegende CMMS eine einfaktorielle Struktur aufweist, wurde eine konfirmatorische Faktorenanalyse mithilfe der Software AMOS 27.0 durchgeführt. Die Daten wurden auf Basis der Kovarianzmatrix der Items und mittels der Maximum Likelihood Methode analysiert. Die Modellgüte wurde gemäß den Empfehlungen von Marsh, Hau und Wen (2004) anhand des Comparative Fit Index (CFI), des Tucker-Lewis Index (TLI), des Root Mean Square Error of Approximation (RMSEA) und des Standardized Root Mean Square Residual (SRMR) beurteilt. Folgende Kriterien wurden dabei für einen guten bzw. akzeptablen Modell-Fit verwendet: CFI und TLI > .95 bzw. .90, RMSEA < .05 bzw. .08 und SRMR < .05 bzw. .10 (Hu und Bentler, 1999). Zur Ermittlung der Kriteriums- 
Tabelle 2. Itemformulierungen, deskriptive Kennwerte, Trennschärfen und Faktorladungen der Items

\begin{tabular}{|c|c|c|c|c|}
\hline Item & M & $S D$ & $r_{\text {it }}$ & $\lambda$ \\
\hline 1. In unserer Trainingsgruppe sind die Mitglieder dazu bereit, die Regeln zu brechen, um im Sport voranzukommen. & 4.15 & 1.02 & .57 & .56 \\
\hline 2. In unserer Trainingsgruppe ist es wichtiger, Leistung zu bringen als ehrlich zu sein. & 4.09 & 1.08 & .72 & .77 \\
\hline 3. In unserer Trainingsgruppe ist Durchsetzungsvermögen wichtiger als Fairness. & 3.98 & 1.07 & .73 & .78 \\
\hline 4. Bei uns zählt Erfolg mehr als Hingabe und Loyalität. & 3.89 & 1.16 & .72 & .76 \\
\hline 5. In unserer Trainingsgruppe ist es wichtiger, erfolgreich zu sein als anderen zu helfen. & 3.95 & 1.13 & .72 & .75 \\
\hline $\begin{array}{l}\text { 6. In unserer Trainingsgruppe streben die Mitglieder nach Erfolg und Ansehen, auch wenn dabei die eigenen Werte } \\
\text { auf der Strecke bleiben. }\end{array}$ & 3.91 & 1.12 & .74 & .79 \\
\hline 7. In unserer Trainingsgruppe sind die Mitglieder bereit zu lügen, um selber im Sport voranzukommen. & 3.96 & 1.17 & .74 & .74 \\
\hline $\begin{array}{l}\text { 8. Die Mitglieder unserer Trainingsgruppe sind dazu bereit, ihre Werte außer Acht zu lassen, wenn sie sich dadurch } \\
\text { einen Vorteil verschaffen können. }\end{array}$ & 4.11 & 1.07 & .78 & .77 \\
\hline
\end{tabular}

Anmerkungen: $N=545 . M$ = Mittelwert, $S D=$ Standardabweichung, $r_{\text {it }}=$ Trennschärfe, $\lambda=$ standardisierte Faktorladung

validität (konkurrente Validität) diente die Berechnung von Regressionsanalysen zur Vorhersage von Dopingabsichten und antisozialem Verhalten durch die KMES. Um die Validität des Konstruktes über ein weiteres Kriterium zu bestimmen, wurden Pearson Korrelationen zwischen der KMES und den Subskalen des PMCSQ-D berechnet.

\section{Ergebnisse}

\section{Item- und Skalenkennwerte und Reliabilität}

Die Mittelwerte und Standardabweichungen der Items der KMES (Gesamtstichprobe $N=545$ ) können Tabelle 2 entnommen werden. Die Schiefe der Items reicht von -1.14 für Item 1 bis -0.78 für Item 4 und die Kurtosis von -0.32 für Item 4 bis 0.57 für Item 2. Die Trennschärfekoeffizienten der Items liegen alle in dem Bereich $.57<r_{\text {it }}<$ .78 (siehe Tab. 2). Die Gesamtskala weist einen Mittelwert von $M=4.01(S D=0.87)$, eine Schiefe von -0.87 und eine Kurtosis von -0.03 auf. Die Sportler_innen nahmen im Durchschnitt eine hohe kollektiv-moralische Einstellung ihrer Gruppe wahr. Die Skalenhomogenitätsprüfung ergibt ein Cronbachs Alpha von $\alpha=.91$. Die Prüfung der Merkmalsstabilität der KMES (Testwiederholung mit $n=$ 63 Personen der Stichprobe 2 nach 6-8 Wochen) offenbart einen Retest-Reliabilitätskoeffizienten von $r_{\mathrm{tt}}=.59, p$ $<.001$.

\section{Faktorenstruktur}

Die Überprüfung der postulierten eindimensionalen Struktur der KMES wurde anhand der Gesamtstichprobe von $N=545$ durchgeführt. Die Fit-Indizes der konfirmatorischen Faktorenanalyse waren wie folgt: $\chi^{2}(20)=$
221.959, $p<.001, \mathrm{CFI}=.920, \mathrm{TLI}=.888, \mathrm{RMSEA}=.138$, $\mathrm{SRMR}=0.049$. Während die Werte des CFI und des SRMR einen akzeptablen Modell-Fit nahelegen, weisen die Werte des TLI und des RMSEA auf eine Diskrepanz zwischen dem postulierten Modell und den Daten der Stichprobe hin. Die Inspektion der Modifikationsindizes zeigte, dass einige Itempaare hohe Residuen aufwiesen, was ein Hinweis auf die Korrelation zwischen den Messfehlern dieser Items ist. Somit wurden die Paare 1-7, 1-8, 7-8 und 4-5 identifiziert und hinsichtlich formulierungsspezifischer und inhaltlicher Auffälligkeiten untersucht (Brown, 2015). Da sich die Items 1,7 und 8 in ihrer Formulierung stark ähneln und diese Formulierung von allen anderen Indikatoren abweicht, ist es plausibel, dass die Messfehler dieser Items korrelieren. Ebenso ist eine Korrelation der Messfehler von Item 4 und 5 vertretbar, da dies die einzigen Items sind, die beide inhaltlich „Erfolg über Hilfsbereitschaft/Loyalität" zum Ausdruck bringen. Aus diesen Gründen wurde eine zweite konfirmatorische Faktorenanalyse, welche die aufgeführten MessfehlerKorrelationen zuließ, durchgeführt. Dadurch konnte der Modell-Fit verbessert werden, sodass nun alle herangezogenen Fit-Indizes eine gute bzw. akzeptable (RMSEA) Passung an die Daten nahelegen $\left(\mathrm{X}^{2}(16)=67.308, p<\right.$ $.001, \mathrm{CFI}=.980, \mathrm{TLI}=.964, \mathrm{RMSEA}=.078, \mathrm{SRMR}=$ 0.028) und somit die einfaktorielle Struktur mit 8 Items bestätigen. Wie in Tabelle 2 dargestellt, laden alle Items mit $.57<\lambda<.79$ hoch auf diesen Faktor.

\section{Kriteriumsvalidität}

Zur Überprüfung der Gültigkeit der KMES über verschiedene Außenkriterien (konkurrente Validität) wurden zunächst lineare Regressionsanalysen mit der kollektiv-moralischen Einstellung als jeweiliger Prädiktor 
für Dopingabsichten und antisoziales Verhalten durchgeführt.

Da Dopingabsichten nur in der Substichprobe 1 erfasst wurde (siehe Tab. 1) beziehen sich die folgenden Ergebnisse auf eine Stichprobengröße von $n=267$. Der Skalenmittelwert $(M=3.84, S D=0.95)$ sowie die interne Konsistenz $(\alpha=.92)$ der KMES fallen ähnlich hoch wie in der Gesamtstichprobe aus. Die Variable Dopingabsichten weist einen Mittelwert von $M=2.18$ ( $S D=$ 1.15) auf und kann von der KMES mit einer Varianzaufklärung von $12.8 \%(F=38.56 ; p<.001)$ vorhergesagt werden $(b=-.43, T=-6.21 ; p<.001)$.

Das antisoziale Verhalten wurde nur in Substichprobe 2 erhoben, weshalb sich die folgenden Ergebnisse auf eine Proband_innenzahl von $n=179$ beziehen. Auch in dieser Stichprobe ähnelt der Skalenmittelwert der KMES $(M=4.34, S D=0.65)$ und der Reliabilitätskoeffizient $(\alpha=.92)$ der Gesamtstichprobe. Den Ergebnissen nach verhalten sich die Sportler_innen im Durchschnitt selten antisozial $(M=1.65, S D=0.59)$. Die KMES erweist sich auch hier in der Lage, das Kriterium vorherzusagen $(b=-.38, T=-6.04 ; p<.001)$. Die Varianzaufklärung liegt bei $17.1 \%(F=36.50 ; p<.001)$.

Eine weitere Validierung des Konstruktes wurde durch die Berechnung der Korrelationen zwischen der KMES und dem PMCSQ-D vorgenommen. Wie antisoziales Verhalten wurde auch das motivationale Trainingsklima nur von der Substichprobe $2(n=179)$ erfasst. Eine Betrachtung der Mittelwerte ergibt, dass die Teilnehmenden ein eher aufgabenorientiertes (AUF, $M$ $=3.92, S D=0.59$ ) als ein wettbewerbsorientiertes (WETT, $M=2.27, S D=0.73$ ) Trainingsklima wahrnehmen. Es zeigt sich für die kollektiv-moralische Einstellung ein signifikant positiver Zusammenhang mit der Skala AUF $(r=.35 ; p<.001)$ und ein signifikant negativer Zusammenhang mit der Skala WETT des PMCSQ$\mathrm{D}(r=-.52 ; p<.001)$. Die Ergebnisse zur kriterialen Validität sowie die Skalenkennwerte der herangezogenen Konstrukte sind in Tabelle 3 veranschaulicht.

\section{Diskussion}

Zielsetzung dieser Untersuchung war es, ein Instrument zur Erhebung der kollektiv-moralischen Einstellung von Gruppen für den deutschsprachigen Sport- und Dopingkontext zu erstellen und dieses Instrument hinsichtlich seiner Testgütekriterien zu überprüfen. Grundlage für die hier vorgestellte Skala bildete die Collective Moral Motivation Scale (Arnaud, 2010) aus der Arbeits- und Organisationspsychologie. Die Ergebnisse der Testgüteprüfung zeigen, dass der entstandene Fragebogen KMES zur Be-
Tabelle 3. Skalenmittelwerte, Standardabweichungen, Reliabilitätskoeffizienten der Konstrukte sowie Regressionsgewichte und Korrelationskoeffizienten

\begin{tabular}{|c|c|c|c|c|c|c|}
\hline & & $M$ & $S D$ & $\alpha$ & $b$ & $r$ \\
\hline \multicolumn{2}{|l|}{ KMES $^{a}$} & 4.01 & 0.87 & .91 & & \\
\hline \multicolumn{2}{|c|}{ Dopingabsichten ${ }^{b}$} & 2.18 & 1.15 & .88 & $-.43 * *$ & \\
\hline \multicolumn{2}{|c|}{ Antisoziales Verhalten ${ }^{c}$} & 1.65 & 0.59 & .91 & $-.38 * *$ & \\
\hline \multirow[t]{2}{*}{$P M C S Q-D^{c}$} & AUF & 3.92 & 0.59 & .75 & & $.35^{\star *}$ \\
\hline & WETT & 2.27 & 0.73 & .83 & & $-.52 * \star$ \\
\hline
\end{tabular}

Anmerkungen: $b=$ Betagewicht der Regressionsanalysen mit der KMES als Prädiktor. $r=$ Korrelationen mit der KMES. ${ }^{* \star} p<.001 .{ }^{a}$ Beruht auf $N=545$. ${ }^{\mathrm{b}}$ Beruht auf $N=267 .{ }^{\circ}$ Beruht auf $N=179$. AUF = Aufgabenorientiertes Trainingsklima; WETT $=$ Wettbewerbsorientiertes Trainingsklima

stimmung der kollektiven moralischen Einstellung eines Teams oder einer Trainingsgruppe geeignet ist.

Die Annahme eines eindimensionalen Konstruktes konnte durch eine konfirmatorische Faktorenanalyse bestätigt werden. Die Fit-Indizes bezeugen einen zufriedenstellenden bis guten Modell-Fit für ein einfaktorielles Modell mit Zulassung von Messfehler-Korrelationen zwischen den Items 1 und 7, 1 und 8, 7 und 8 sowie zwischen den Items 4 und 5. Die Items 1, 7 und 8 sind in ihrer Formulierung sehr ähnlich und unterscheiden sich darin von allen weiteren Items, wodurch die gemeinsame Varianz ihrer Messfehler erklärt werden kann. Da sie inhaltlich drei verschiedene Aspekte der kollektiv-moralischen Einstellung widerspiegeln (Item 1: die Regeln brechen, Item 7: lügen, Item 8: die Werte außer Acht lassen), sollten alle drei Items beibehalten werden. Im Gegensatz dazu scheint bei Item 4 und 5 eine inhaltliche Ähnlichkeit vorzuliegen. Beide Items drücken den Aspekt „Erfolg über Loyalität und Hingabe (Item 4) bzw. Hilfsbereitschaft (Item5)“ aus. Hier wäre in zukünftigen Studien zu prüfen, ob eines der beiden Items gelöscht werden könnte, um die Gesamtitemzahl zu verringern. Für die vorläufige Validierung wurde jedoch zugunsten der Vergleichbarkeit mit der ursprünglichen Skala aus dem Arbeitskontext auf die Löschung von Items verzichtet. Im Rahmen der faktorenanalytischen Prüfung kritisch anzumerken ist die Verwendung der Maximum Likelihood Methode zur Schätzung des Modell-Fits, die eine parametrische Verteilung der Daten voraussetzt. Wenn auch die absoluten Werte der univariaten Schiefe und Kurtosis in einem akzeptablen Bereich liegen, wäre aufgrund der Verletzung der multivariaten Normalverteilung auch eine robustere Schätzmethode in Betracht zu ziehen, welche dem Modell mehr Stabilität geben könnte (Li, 2016).

Die interne Konsistenz des Fragebogens erwies sich mit einem Cronbachs Alpha von .91 als exzellent, während die Korrelation zwischen Test und Testwiederholung auf eine geringere, aber dennoch ausreichende Merkmalssta- 
bilität hindeutet. Die einzelnen Items erwiesen sich mit Werten von $r_{\text {it }}>.50$ als trennscharf. $\mathrm{Zu}$ beachten ist hierbei allerdings, dass alle Trennschärfekoeffizienten mit der Ausnahme von Item 1 sogar den Wert .70 überschreiten. Dies trifft ebenfalls auf die Faktorladungen zu und könnte auf eine starke inhaltliche Ähnlichkeit und die potentielle Redundanz einiger Items hinweisen. Die hohen Trennschärfen könnten aber auch daher rühren, dass ähnliche Itemformulierungen zu einer lokalen Abhängigkeit führen (Beauducel \& Leue, 2014), wodurch wiederum auch die interne Konsistenz begünstigt werden könnte. Zukünftige Studien sollten daher, wie bereits weiter oben angesprochen, eine Item-Reduktion prüfen.

Neben dem Erfüllen der Reliabilität und der faktoriellen Validität liefern die Untersuchungen zur kriterienbezogenen konkurrenten Validität Hinweise für die Gültigkeit der KMES. Da sich in vorherigen Studien moralische Normen und die moralische Atmosphäre eines Teams als Prädiktoren für Dopingabsichten herausstellten (Kavussanu et al., 2015; Ntoumanis et al., 2014), sollte auch die kollektiv-moralische Einstellung einer Trainingsgruppe das genannte Kriterium zu einem gewissen Grad vorhersagen können. Sportler_innen aus einer Trainingsgruppe, in welcher moralische Werte wie Fairness Priorität gegenüber anderen Werten wie Erfolg um jeden Preis haben, sollte eher geringere Dopingabsichten aufweisen. Tatsächlich erwies sich die KMES als negativer Prädiktor für Dopingabsichten und konnte zur Varianzaufklärung beitragen. Trotz der gefundenen Zusammenhänge ist anzumerken, dass, gerade bei solch sensiblen Variablen wie Dopingabsichten sozial erwünschtes Antworten nicht vollständig kontrolliert werden kann. Der Vorteil der verwendeten indirekten Fragetechnik durch fiktive Szenarien ist aber, dass damit stellvertretend Verhaltensabsichten erfasst werden können und möglicherweise ehrlichere Antworten als bei der direkten Frage nach Doping erhalten werden können (Lazuras, Barkoukis, Rodafinos \& Tzorbatzoudis, 2010). Die größte Varianzaufklärung konnte die KMES bei dem hinzugezogenen Kriterium antisoziales Verhalten leisten. Dieser Befund geht mit den Ergebnissen von Spruit et al. (2019) einher, dass sich Personen einer Trainingsgruppe mit einem hohen moralischen Klima weniger antisozial verhalten und bestätigt damit ebenfalls die Validität der KMES. Als weitere Untermauerung zur Validität des Konstruktes bildete die Skala den empirisch nachgewiesenen und damit theoretisch erwarteten $\mathrm{Zu}$ sammenhang zum motivationalen Trainingsklima ab. Dieses Konstrukt wird anhand des PMCSQ-D (Würth et al., 1999) gemessen und kann zwei Formen annehmen. Bei einem wettbewerbsorientierten Klima steht das Gewinnen und Übertreffen anderer im Vordergrund und nur den Besten im Team wird die größte Beachtung geschenkt. Ein aufgabenorientiertes Trainingsklima hinge- gen herrscht vor, wenn in erster Linie auf die Verbesserung der individuellen Fertigkeiten geachtet wird und die Anstrengung der einzelnen Sportler_innen toleriert und gelobt wird (Seifriz et al., 1992). Die Art des motivationalen Klimas reflektiert auch die Werte und Prioritäten der Gruppe, die nicht selten von Trainer_innen ausgehen (Kavussanu und Spray, 2006). In einer Trainingsgruppe, in der nach dem Motto „Gewinnen ist Alles“ trainiert und gehandelt wird, ist es wahrscheinlicher, dass Werte wie Respekt gegenüber den Regeln oder gegenüber den Opponenten vernachlässigt werden und Betrug und Aggression als legitim angesehen wird, um das Hauptziel des Gewinnens und Übertreffens zu erreichen (Ommundsen et al., 2003). Demgegenüber ist es in einer Trainingsgruppe, in der auf die individuellen Fertigkeiten jedes einzelnen eingegangen wird und Gewinnen nicht an erster Stelle steht, eher wahrscheinlich, dass geteilte Normen entstehen, die moralisches Verhalten priorisieren (höhere Werte der KMES). Wie erwartet, korrelierte ein wettbewerbsorientiertes Trainingsklima signifikant negativ mit der KMES. Im Gegensatz dazu zeigte sich ein signifikant positiver Zusammenhang der KMES mit einem aufgabenorientierten Trainingsklima.

Wenn auch die psychometrischen Eigenschaften der Skala KMES sehr zufriedenstellend ausfallen, sind die folgenden Aspekte kritisch zu bedenken: Die Ausprägung der gemessenen Variablen zeigt, dass die Proband_innen im Durchschnitt, erstens, eine hohe kollektiv-moralische Einstellung in ihren Trainingsgruppen wahrnehmen, zweitens, sehr geringe Dopingabsichten haben, und drittens, sich selten antisozial verhalten. Demzufolge können wir schlussfolgern, dass hohe kollektive moralische Normen zwar mit geringen Dopingabsichten und einem geringen antisozialen Verhalten zusammenhängen; wir können allerdings nicht daraus schließen, ob eine geringere kollektiv-moralische Einstellung tatsächlich mit hohen Dopingabsichten und häufigem antisozialen Verhalten einhergeht. Wie bei zahlreichen Studien in diesem Forschungsfeld wurden auch in der vorliegenden Arbeit lediglich die positiven Einstellungen und Verhaltensweisen abgebildet, wodurch die Frage unbeantwortet bleibt, welche Faktoren tatsächlich mit unmoralischem Verhalten wie Doping einhergehen. Dennoch bieten Skalen wie die hier vorgestellte einen entscheidenden Wert für die Erforschung moralrelevanten Verhaltens, da sie uns aufzeigen, welche Faktoren beispielsweise mit „NichtDopen" assoziiert sind und demzufolge in Präventionsmaßnahmen adressiert und gefördert werden sollten. Allerdings gilt es in zukünftigen Studien zu belegen, dass der gefundene Zusammenhang zwischen einer hoch ausgeprägten moralischen Gruppeneinstellung und geringen Dopingabsichten bzw. einem geringen 
antisozialen Verhalten tatsächlich kausal ist und keine Scheinkorrelation darstellt. Um den systematischen Einfluss von potentiellen weiteren Variablen herauszufiltern, sind deshalb partielle Korrelationsanalysen wichtige weitere Schritte in dieser Forschungsrichtung.

Weiterhin ist anzumerken, dass auch bei dem vorliegenden Instrument das Problem der Erforschung von gruppenbezogenen Konstrukten aus Sicht einer Person zutrifft. Die KMES erfasst die von einer Person wahrgenommene moralische Einstellung der Gruppe und erlaubt letztendlich keinen Rückschluss, ob dies auch mit der tatsächlichen Gruppeneinstellung übereinstimmt. Vielmehr könnte eine solche Skala auch die eigenen Einstellungen einer Person oder deren Erwartungen an die Gruppe widerspiegeln. In der Tat wird diese Methode beispielsweise im Dopingkontext genutzt, um die Einstellung auf persönlicher Ebene indirekt zu erfassen bzw. einzuschätzen. Um diesen Aspekt zu kontrollieren, sollten in anschließenden Studien die individuellen Antworten aller Personen, die zu einer Gruppe gehören, aggregiert werden und Variationskoeffizienten für jede Gruppe gebildet werden (vgl. Kavussanu \& Spray, 2006). So könnte man prüfen, inwieweit die Gruppenmitglieder tatsächlich die gleichen moralischen Normen wahrnehmen.

Die dargestellten Befunde in der vorliegenden Arbeit beziehen sich auf eine sehr junge Stichprobe (Durchschnittsalter 18 Jahre), weshalb weiterhin zu testen wäre, ob sich die Ergebnisse auch in einer Stichprobe älterer Personen replizieren lassen. Außer Frage steht jedoch die Relevanz moralbezogener Forschung im Nachwuchs(leistungs)sport, da Sportler_innen in der Adoleszenz beginnen, deviantes Verhalten zu zeigen (Kavussanu, Seal \& Phillips, 2006) und zudem das Verhalten und die Einstellungen in diesem Alter geformt werden - ganz besonders durch das Trainingsumfeld.

Zukünftige Studien sollten den Fragebogen KMES erneut an einer größeren Stichprobe testen, um dessen Validität zu untermauern. Dabei sollten die Zusammenhänge zu den herangezogenen Kriterien vor allem auf latenter Ebene analysiert werden. Darüber hinaus sind weitere Überprüfungen zur konvergenten und divergenten Validität notwendig, um das nomologische Netz um die kollektiv-moralische Einstellung auszubauen. Weiterhin sollte die Skala hinsichtlich ihrer Messinvarianz gegenüber Sportarten und dem Geschlecht untersucht werden.

Zusammenfassend lässt sich festhalten, dass die KMES als erstes Instrument zur Erhebung moralischer Werte auf kollektiver Ebene im deutschsprachigen Raum geeignet ist. Sie stellt eine Möglichkeit dar, valide und reliabel die wahrgenommene Priorität ethischer und moralischer Werte wie Fairness oder Ehrlichkeit gegenüber anderen Werten wie Erfolg oder Ansehen in einer Sportgruppe zu analysieren. Einen besonderen Nutzen bietet die Skala in der Dopingprävention, da sie in der Forschung zu protektiven und Risikofaktoren im Hinblick auf Dopingverhalten neue Erkenntnisse generieren könnte (Erickson, McKenna \& Backhouse, 2015). Sollte sich, beispielsweise, in längsschnittlichen Studien herausstellen, dass ethische und moralische Werte auf Gruppenebene protektiv gegen positive Einstellungen zu Doping bzw. Dopingverhalten wirken, so könnte die Ausbildung dieser Werte zukünftig Bestandteil von Curricula (Aus- und Fortbildungen) für Trainer_innen sein. Vor allem aber in Dopingpräventionsprogrammen, die auf die (Nachwuchs-)Athlet_innen selbst abzielen und die gemäß den Richtlinien des International Standard for Education (WADA, 2021) einen werte-basierten Ansatz verfolgen, könnte die kollektiv-moralische Einstellung der Gruppe gezielt adressiert und mittels der KMES evaluiert werden. Auch in Forschungsprojekten ohne dopingspezifischen Hintergrund kann die KMES eingesetzt werden, um bei moralrelevanten Fragestellungen Aufschluss über die Rolle der Werte auf Teamebene zu erlangen und anschließend gegebenenfalls moralerzieherische Interventionen im Bereich des Teamklimas zu gestalten.

\section{Literatur}

Ajzen, I. (1985). From intentions to actions: A theory of planned behavior. In J. Kuhl \& J. Beckmann (Hrsg.), Action Control (S. 11 -39). Heidelberg: Springer.

Ames, C. (1992). Achievement goals and the classroom motivational climate. Student Perceptions in the Classroom, 1, $327-348$.

Arnaud, A. (2010). Conceptualizing and measuring ethical work climate: Development and validation of the ethical climate index. Business \& Society, 49, $345-358$. https://doi.org/10.1177/ 0007650310362865

Bandura, A. (1991). Social cognitive theory of self-regulation. Organizational Behavior and Human Decision Processes, 50, 248 287. https://doi.org/10.1016/0749-5978(91)90022-L

Beauducel, A. \& Leue, A. (2014). Psychologische Diagnostik (Vol. 19). Göttingen: Hogrefe.

Boardley, I. D., Grix, J. \& Dewar, A. J. (2014). Moral disengagement and associated processes in performance-enhancing drug use: a national qualitative investigation. Journal of Sports Sciences, $32,836-844$.

Brown, T. A. (2015). Confirmatory factor analysis for applied research. New York, NY: Guilford.

Bühner, M. (2011). Einführung in die Test- und Fragebogenkonstruktion (3rd ed.). München: Pearson.

Donovan, R. J., Egger, G., Kapernick, V. \& Mendoza, J. (2002). A conceptual framework for achieving performance enhancing drug compliance in sport. Sports Medicine, 32, 269-284. htt ps://doi.org/10.2165/00007256-200232040-00005 
Erickson, K., McKenna, J. \& Backhouse, S. H. (2015). A qualitative analysis of the factors that protect athletes against doping in sport. Psychology of Sport and Exercise, 16, 149 -155. https:// doi.org/10.1016/j.psychsport.2014.03.007

Guivernau, M. \& Duda, J. L. (2002). Moral atmosphere and athletic aggressive tendencies in young soccer players. Journal of Moral Education, 31, 67 -85. https://doi.org/10.1080/030572401201 11445

Harkness, J. A. (2003). Questionnaire translation. Cross-cultural Survey Methods, 1, 35-56.

Hu, L. T. \& Bentler, P. M. (1999). Cutoff criteria for fit indexes in covariance structure analysis: Conventional criteria versus new alternatives. Structural Equation Modeling: A Multidisciplinary Journal, 6 (1), $1-55$.

Kavussanu, M. \& Boardley, I. D. (2009). The prosocial and antisocial behavior in sport scale. Journal of Sport and Exercise Psychology, 31, 97 -117. https://doi.org/10.1037/t33475-000

Kavussanu, M., Elbe, A. M. \& Hatzigeorgiadis, A. (2015). A Crosscultural Approach to a Cross-cultural Issue: Psychosocial Factors and Doping in Young Athletes: Final Report for the World Anti-Doping Agency, Social Science Research Grant (20132015). University of Birmingham.

Kavussanu, M., Roberts, G. C. \& Ntoumanis, N. (2002). Contextual influences on moral functioning of college basketball players. The Sport Psychologist, 16, 347-367. https://doi.org/10.1123/ tsp.16.4.347

Kavussanu, M., Seal, A. R. \& Phillips, D. R. (2006). Observed prosocial and antisocial behaviors in male soccer teams: Age differences across adolescence and the role of motivational variables. Journal of Applied Sport Psychology, 18, 326-344. htt ps://doi.org/10.1080/10413200600944108

Kavussanu, M. \& Spray, C. M. (2006). Contextual influences on moral functioning of male youth footballers. The Sport Psychologist, 20, 1 - 23. https://doi.org/10.1123/tsp.20.1.1

Kavussanu, M., Stanger, N. \& Boardley, I. D. (2013). The Prosocial and Antisocial Behaviour in Sport Scale: Further evidence for construct validity and reliability. Journal of Sports Sciences, 31, $1208-1221$.

Kavussanu, M., Yukhymenko-Lescroart, M. A., Elbe, A. M. \& Hatzigeorgiadis, A. (2020). Integrating moral and achievement variables to predict doping likelihood in football: A cross-cultural investigation. Psychology of Sport and Exercise, 47, 101518. htt ps://doi.org/10.1016/j.psychsport.2019.04.008

Kline, R. B. (2005). Methodology in the social sciences. Principles and practice of structural equation modeling (2nd ed.). New York, NY: Guilford.

Lazuras, L., Barkoukis, V., Mallia, L., Lucidi, F. \& Brand, R. (2017). More than a feeling: The role of anticipated regret in predicting doping intentions in adolescent athletes. Psychology of Sport and Exercise, 30, 196-204. https://doi.org/10.1016/j.psychs port.2017.03.003

Lazuras, L., Barkoukis, V., Rodafinos, A. \& Tzorbatzoudis, H. (2010). Predictors of doping intentions in elite-level athletes: a social cognition approach. Journal of Sport and Exercise Psychology, 32, 694-710.

Li, C. H. (2016). Confirmatory factor analysis with ordinal data: Comparing robust maximum likelihood and diagonally weighted least squares. Behavior Research Methods, 48, 936 -949. htt ps://doi.org/10.3758/s13428-015-0619-7

Marsh, H. W., Hau, K. T. \& Wen, Z. (2004). In search of golden rules: Comment on hypothesis-testing approaches to setting cutoff values for fit indexes and dangers in overgeneralizing $\mathrm{Hu}$ and Bentler's (1999) findings. Structural Equation Modeling, 11, $320-341$.

Ntoumanis, N., Ng, J. Y., Barkoukis, V. \& Backhouse, S. (2014). Personal and psychosocial predictors of doping use in physical activity settings: A meta-analysis. Sports Medicine, 44, 1603 1624.

Ntoumanis, N., Taylor, I. M. \& Thøgersen-Ntoumani, C. (2012). A longitudinal examination of coach and peer motivational climates in youth sport: Implications for moral attitudes, wellbeing, and behavioral investment. Developmental Psychology, 48, $213-223$.

Ommundsen, Y., Roberts, G. C., Lemyre, P. N. \& Treasure, D. (2003). Perceived motivational climate in male youth soccer: Relations to social-moral functioning, sportspersonship and team norm perceptions. Psychology of Sport and Exercise, 4, 397 - 413. htt ps://doi.org/10.1016/S1469-0292(02)00038-9

Petróczi, A. \& Aidman, E. (2008). Psychological drivers in doping: The life-cycle model of performance enhancement. Substance Abuse Treatment, Prevention, and Policy, 3, 7. https://doi.org/ 10.1186/1747-597X-3-7

Power, F. C., Higgins, A. \& Kohlberg, L. (1989). Lawrence Kohlberg's approach to moral education. New York, NY: Columbia University Press.

Ring, C., Kavussanu, M. \& Gürpınar, B. (2020). Basic values predict doping likelihood. Journal of Sports Sciences, 38, 357-365. htt ps://doi.org/10.1080/02640414.2019.1700669

Schwartz, S. H. (1992). Universals in the content and structure of values: Theoretical advances and empirical tests in 20 countries. Advances in Experimental Social Psychology, 25, 1 - 65.

Schwartz, S. H. (2012). An overview of the Schwartz theory of basic values. Online readings in Psychology and Culture, 2 (1). https:// doi.org/10.9707/2307-0919.1116

Seifriz, J., Duda, J. L. \& Chi, L. (1992). The relationship of perceived motivational climate to achievement-related affect and cognitions in basketball. Journal of Sport \& Exercise Psychology, 14, $375-391$.

Shields, D. L. L., Gardner, D. E., Bredemeier, B. J. L. \& Bostrom, A. (1995). Leadership, cohesion, and team norms regarding cheating and aggression. Sociology of Sport Journal, 12, 324-336.

Sohnsmeyer, J. (2018). Deutsche Adaptation der Prosocial and Antisocial Behavior in Sport Scale (PABSS). Diagnostica, 64, 180 - 190. https://doi.org/10.1026/0012-1924/a000206

Spruit, A., Kavussanu, M., Smit, T. \& IJntema, M. (2019). The relationship between moral climate of sports and the moral behavior of young athletes: A multilevel meta-analysis. Journal of Youth and Adolescence, 48, 228 - 242. https://doi.org/10.1007/ s10964-018-0968-5

Stanger, N., Backhouse, S. H., Jennings, A. \& McKenna, J. (2018). Linking motivational climate with moral behavior in youth sport: The role of social support, perspective taking, and moral disengagement. Sport, Exercise, and Performance Psychology, 7, 392 - 407. https://doi.org/10.1037/spy0000122

Stephens, D. E. (2000). Predictors of likelihood to aggress in youth soccer: An examination of coed and all-girls teams. Journal of Sport Behavior, 23(3), 311-315.

Stephens, D. E. (2001). Predictors of aggressive tendencies in girls' basketball: An examination of beginning and advanced participants in a summer skills camp. Research Quarterly for Exercise and Sport, 72, $257-266$.

Stephens, D. E. (2004). Moral atmosphere and aggression in collegiate intramural Sport. International Sports Journal, 8(1), $65-75$.

van de Pol, P. K., Kavussanu, M. \& Claessens, B. (2020). Moral functioning across training and competition in sport. International Journal of Sport and Exercise Psychology, 18, 239-255. https://doi.org/10.1080/1612197X.2018.1511623

World Anti-Doping Agency (WADA). (2021, Januar). WADA International Standard for Education. Verfügbar unter: https://www. wada-ama.org/sites/default/files/resources/files/internatio nal_standard_ise_2020.pdf 
Würth, S., Saborowski, C. \& Alfermann, D. (1999). Trainingsklima und Führungsverhalten aus der Sicht jugendlicher Athleten und deren Trainer. Psychologie und Sport, 6, 146-157.

Zelli, A., Mallia, L. \& Lucidi, F. (2010). The contribution of interpersonal appraisals to a social-cognitive analysis of adolescents' doping use. Psychology of Sport and Exercise, 11, 304-311. htt ps://doi.org/10.1016/j.psychsport.2010.02.008

\section{Förderung}

Diese Arbeit wurde mit Forschungsmitteln des Bundesinstituts für Sportwissenschaften aufgrund eines Beschlusses des Deutschen Bundestages gefördert (AZ: ZMVI4-070301/19-21).

Open Access-Veröffentlichung ermöglicht durch die Universität Leipzig.

\section{ORCID}

Theresa Manges

(iD) https://orcid.org/0000-0001-6088-8927

\section{Nadja Walter}

Universität Leipzig

Sportwissenschaftliche Fakultät

Institut für Sportpsychologie und Sportpädagogik

Jahnallee 59

04109 Leipzig

nadja.walter@uni-leipzig.de 